\section{A Wavelet-Balance Approach for Steady-State Analysis of Nonlinear Circuits}

\author{
Xin Li, Bo Hu, Xieting Ling, and Xuan Zeng
}

\begin{abstract}
In this paper, a novel wavelet-balance method is proposed for steady-state analysis of nonlinear circuits. Taking advantage of the superior computational properties of wavelets, the proposed method presents several merits compared with those conventional frequency-domain techniques. First, it has a high convergence rate $O\left(h^{4}\right)$, where $h$ is the step length. Second, it works in time domain so that many critical problems in frequency domain, such as nonlinearity and high order harmonics, can be handled efficiently. Third, an adaptive scheme exists to automatically select proper wavelet basis functions needed at a given accuracy. Numerical experiments further prove the promising features of the proposed method in solving steady-state problems.
\end{abstract}

Index Terms-Nonlinear circuit, steady-state analysis, wavelet balance.

\section{INTRODUCTION}

A major difficulty in time-domain simulation of nonlinear circuits, such as power supplies, high- $Q$ amplifiers, modulators and oscillators, etc., is that the transient response may stand for quite a long time before the steady-state is reached. This problem makes it infeasible to calculate the steady-state response by conventional transient simulation algorithms because direct integration of the circuit equations throughout the transients consumes unbearable computing time.

During the past several decades, a great number of techniques have been developed to solve the periodic steady-state problem [1]-[11], which may be categorized into three classes: shooting methods [1]-[4], harmonic balance methods [5]-[10] and sample balance methods [11]. The shooting methods attempt to find a set of initial conditions satisfying the two-point boundary constraint, such that the circuit starts in periodic steady state directly. However, the shooting methods consume expensive computing time since they require to numerically integrate the system equations time after time. The harmonic balance methods assume the circuit solutions in the form of Fourier series. Moreover, they divide the circuit into a linear and a nonlinear part so that the linear subnetwork could be solved efficiently in frequency domain. Unfortunately, the harmonic balance methods need to repeatedly execute DFT and IDFT operations during the solution process, and employ a large number of harmonic components to achieve an accurate simulation result. Therefore, they also expend substantial computing time. The sample balance methods directly approximate the time-domain state-variable waveforms by suitable basis functions, such as periodic cubic splines, and use time-domain samples as problem unknowns. Nevertheless, there doesn't exist a strong theory to ensure the convergence features of these methods.

Recently, the wavelet theory has been well developed [12], [13] and widely used in many applications, such as solving partial differential equations [14], [15] and performing high-speed circuit simulations [16], [17]. However, the wavelet-based method for steady-state

Manuscript received April 25, 2000; revised April 11, 2001. This work was supported in part by the National Science Foundation of China (NSFC ) under Grant 69806004, Grant 69928402, and Grant 60176017, in part by the Foundation for University Key Teacher, Ministry of Education, in part by the Doctoral Program Foundation, Ministry of Education of China under Grant 2000024628, in part by the Shanghai Science and Technology Committee under Project 01JC14014 and in part by the Shanghai AM R\&D under Funding 0107. This paper was recommended by Associate Editor M. Gilli.

The authors are with the ASIC \& System State Key Laboratory, Department of Electronics Engineering, Fudan University, Shanghai 200433, China.

Publisher Item Identifier S 1057-7122(02)04724-4. analysis has never been explored. In this paper, we propose a novel wavelet-based algorithm, named the wavelet-balance method, to access the periodic steady-state problem. Taking advantage of the wavelets, the proposed method works more efficiently in time domain than those frequency-domain techniques, and has a solid theoretical background based on wavelet approximation theory.

The rest of this paper is organized as follows. In Section II, we first introduce the basic principle of the wavelet-balance method for steady-state analysis, then compare the proposed algorithm with conventional Fourier-based techniques [5]-[10] in Section III. Numerical experiments are presented in Section IV to demonstrate the computational efficiency of the proposed algorithm. Finally, we draw conclusions in Section V and outline some important background of the wavelet basis functions in the Appendix.

\section{WAVELET BALANCE APPROACH FOR STEADY-STATE ANALYSIS}

\section{A. Principle of Wavelet Balanced Steady-State Analysis}

Without loss of generality, we assume that a circuit is described by an ordinary differential equation of the type

$$
\frac{d X}{d t}=f(X, t)
$$

where $\left[X(t)=X_{1}(t) X_{2}(t) \cdots X_{N}(t)\right]^{T}$ are the $N$ unknown state variables, and $f(X, t)$ is a given nonlinear vector function.

The basic idea of the wavelet-balance method in this paper is to expand the unknown state variables by wavelet series, but not the Fourier series that have been used in the conventional techniques [5]-[10]. Assume the steady-state response period of the circuit is $T$, and the Sobolev space $H^{2}[0, L]$ is studied for wavelet expansion. Usually, because the period $T$ doesn't satisfy some specific conditions needed for wavelets, for example, $L \geq 4$ corresponding to the wavelet basis functions in [16], [17], a scaling operation should be applied to map the interval $[0, T]$ to $[0, L]$, i.e.,

$$
l=K \cdot t
$$

where $t \in[0, T], l \in[0, L]$, and $K$ is a constant which equals to $L / T$. Substituting (2) into (1), we obtain

$$
K \cdot \frac{d X}{d l}=f\left(X, \frac{l}{K}\right) .
$$

In order to find the steady-state solutions, the state variables $X(l)$ are expanded by wavelets

$$
\begin{aligned}
X(l)= & {\left[\begin{array}{c}
X_{1}(l) \\
X_{2}(l) \\
\vdots \\
X_{N}(l)
\end{array}\right] } \\
= & {\left[\begin{array}{cccc}
C_{11} & C_{12} & \cdots & C_{1 M} \\
C_{21} & C_{22} & \cdots & C_{2 M} \\
\vdots & \vdots & \vdots & \vdots \\
C_{N 1} & C_{N 2} & \cdots & C_{N M}
\end{array}\right] \cdot\left[\begin{array}{c}
B_{1}(l) \\
B_{2}(l) \\
\vdots \\
B_{M}(l)
\end{array}\right] } \\
= & C \cdot B(l)
\end{aligned}
$$

where $C \in R^{N \times M}$ is the coefficient matrix, $\left\{B_{i}(l), i=\right.$ $1,2, \ldots, M\}$, are wavelet basis functions, and $M$ is the total number of basis functions that have been employed. The wavelet basis functions can be constructed by many means [13], but in this paper, we prefer to use the basis functions in [16], [17] because they are 
proved to have a high convergence rate $O\left(h^{4}\right)$, where $h$ is the step length [16], [17].

Substituting (4) into (3), we get

$$
K \cdot C \cdot \frac{d B(l)}{d l}=f\left(C B(l), \frac{l}{K}\right) .
$$

Then, discretize the state (5) at some interior collocation points $\left\{l_{1}, l_{2}, \ldots, l_{M}\right\}[16],[17]$ as shown in (6) at the bottom of the page. Equations (1) to (6), are similar to those illustrated in [16] and [17]. But for steady-state response, the state variables $X(l)$ should further satisfy the two-point boundary constraint

$$
X(0)=X(L) .
$$

Thus, we get one more vector equation for the coefficient matrix $C$

$$
C \cdot B(0)=C \cdot B(L) \text {. }
$$

For nonautonomous circuits, the steady-state response period $T$ is determined by input excitations, and the constant $K$ in (6) is known in advance. Hence, there are $M \cdot N$ problem unknowns in coefficient matrix $C$ to be determined. Meantime, the total number of independent algebraic equations in (6) and (8) is $(M+1) \cdot N$. In order to solve these equations, we define the merit function shown in (9) at the bottom of the page where $\|\cdot\|$ denotes the Frobenius norm. Employing the optimization algorithm, such as the Levenberg-Marquardt method in [18], to minimize the merit function $Q$, we can obtain the least-squareerror solution $C$, as well as the steady-state response

$$
\begin{aligned}
& X(l)=C \cdot B(l) \\
& X(t)=C \cdot B(K t) .
\end{aligned}
$$

On the other hand, in autonomous cases, there are totally $M \cdot N+1$ unknown variables, since the oscillation period $T$ and consequently the constant $K$ in (6) are undeterminated. Recall that the overall number of independent algebraic equations in (6) and (8) is $(M+N) \cdot N$, which is greater than or at least equals to the number of problem unknowns. Therefore, the same optimization algorithm can be used to minimize the merit function (9) and find the least-square-error solutions of coefficient matrix $C$ and the unknown parameter $K$.

In summary, the essence of the proposed wavelet-balance method is to approximate the state variables by wavelet basis functions, but not the Fourier basis $e^{j 2 \pi k / T}$ as in many conventional algorithms. Moreover, the wavelets are forced to keep "balance" at the two boundary points in (8), so that the steady-state solution is guaranteed when the state equation is solved.

\section{B. Adaptive Technique}

One of the main advantages of the wavelet-balance method is that there exists an adaptive scheme, which relies on the multiresolution analysis in wavelet theory [15]-[17]. Using adaptive techniques, those wavelet basis functions, which are needed for approximating state variables, can be employed automatically. It, in turn, improves the computational efficiency significantly.

A multiresolution approximation of $H^{2}[0, L]$ is a sequence $\left\{V_{J}, J=\ldots,-1,0,1, \ldots\right\}$ of closed subspaces of $H^{2}[0, L]$

1) $\cdots \subset V_{-1} \subset V_{0} \subset V_{1} \subset \cdots$

2) $\cap_{J=-\infty}^{+\infty} V_{J}=\{0\}$

3) $\left.\cup_{J=-\infty}^{+\infty} V_{J}=H^{2} 0, L\right]$

4) $V_{J}=V_{J-1} \oplus W_{J}$

where the notation $\oplus$ stands for the direct sum. It is clear that the approximation accuracy depends on the wavelet space level $J$. The higher the space level is, the less the error will be. As pointed out in [15]-[17], the magnitude of the wavelet coefficients in $W_{J}$ will indicate whether a refinement, by increasing the wavelet space level, is needed or not. For example, define the maximum relative magnitude of the wavelet coefficients in $W_{J}$ as

$$
R_{J}=\frac{\operatorname{MAX}\left|C_{i}^{J}\right|}{\operatorname{MAX}\left|C_{i}\right|}
$$

where $\operatorname{MAX}\left|C_{i}^{J}\right|$ is the maximum magnitude of the wavelet coefficients in $W_{J}$, and MAX $\left|C_{i}\right|$ is the maximum magnitude of all wavelet coefficients. If $R_{J}$ is greater than a given error tolerance $\varepsilon$, we increase the wavelet space level $J$ to $J^{\prime}$, where $J^{\prime}>J$ [17].

More importantly, because of the compact support of wavelet bases, not all wavelet basis functions in higher wavelet spaces $W_{J^{\prime}}$ are needed in order to achieve more accuracy. In fact, only basis functions, whose positions near the singularities (which can be determined by the magnitude of the wavelet coefficients on level $J$ ), shall be included [17].

\section{COMPARISON With CONVENTIONAL FOURIER-BASED TECHNIQUES}

The key feature of the wavelet-balance method is the fact that wavelet basis has compact support or local support in time domain, whereas Fourier basis $e^{j 2 \pi k / T}$ has a global support. Hence, the proposed wavelet-balance method presents following advantages.

First, the wavelet-balance method has a high-convergence rate, resulting in low computational complexity. It is demonstrated in [16], [17] that the wavelet basis functions presented there, which are also used in this paper, have a high convergence rate $O\left(h^{4}\right)$, where $h$ is the step length, i.e., the distance between two adjacent collocation points.

Second, the wavelet-balance method works in the time domain, so that many critical problems in frequency domain, such as nonlinearity and high-order harmonics, can be handled efficiently. It is well known that when the circuit response is highly distorted by strong nonlinearities, the use of Fourier series with a large number of harmonics may cause considerable computation time, owing to the great number of unknown variables [11].

Third, but most importantly, the adaptive scheme introduced in Section II.B can automatically select proper wavelet basis functions needed

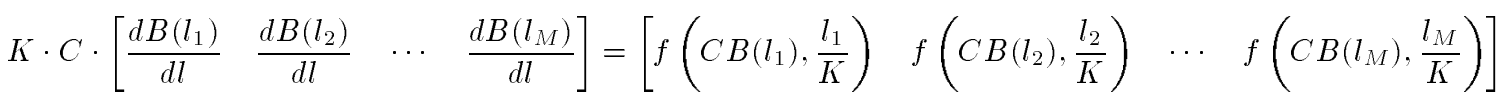

$$
Q=\left\|C B(0)-C B(L) \quad K \cdot C \frac{d B\left(l_{1}\right)}{d l}-f\left(C B\left(l_{1}\right), \frac{l_{1}}{K}\right) \quad \cdots \quad K \cdot C \frac{d B\left(l_{M}\right)}{d l}-f\left(C B\left(l_{M}\right), \frac{l_{M}}{K}\right)\right\|^{2}
$$




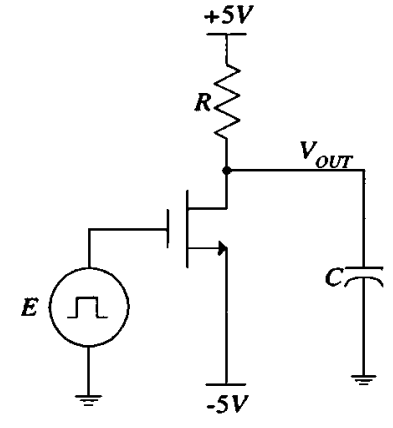

Fig. 1. MOS amplifier where $R=\mathbf{1} \boldsymbol{\Omega}$ and $C=0.1 \mu \mathrm{F}$.

TABLE I

SimUlation Results of the Fourier EXPANSION FOR MOS AMPLIFIER

\begin{tabular}{c|c}
\hline $\begin{array}{c}\text { Basis Function } \\
\text { Number }\end{array}$ & $\begin{array}{c}\text { Relative Simulation Error for } \\
V_{\text {out }}\end{array}$ \\
\hline 21 & $9.606031 \times 10^{-2}$ \\
41 & $5.034534 \times 10^{-2}$ \\
81 & $2.481495 \times 10^{-2}$ \\
161 & $1.155528 \times 10^{-2}$ \\
321 & $4.832508 \times 10^{-3}$ \\
641 & $1.463385 \times 10^{-3}$ \\
\hline
\end{tabular}

at a given accuracy. High-level wavelet basis functions are only employed near singularities. Actually, the response of nonlinear circuits will exhibit localized singularities, which can be easily captured by the wavelet approach through adaptivity.

In addition, it is reported in [5]-[11] that the entire nonlinear network can be decomposed into a linear and a nonlinear multiport subnetwork with the same number of ports. Because the linear part can be solved in frequency domain, computation efficiency can be achieved by choosing the required number of state variables as the same number of linear subnetwork ports, no matter what the actual number of the dynamic elements is. This idea can also be applied to the proposed waveletbalance method, but the detailed implementation is beyond the scope of this paper and will not be presented here.

\section{NUMERICAL EXPERIMENTS}

In this section, three circuit examples have been examined to demonstrate the effectiveness of our proposed wavelet-balance method for steady-state analysis. All of the circuit examples are simulated on a Pentium III-550 computer.

\section{A. MOS Amplifier}

Shown in Fig. 1 is a very simple MOS amplifier from which we can make a full comparison between the proposed wavelet-balance method and those conventional Fourier-based techniques. We test the circuit in Fig. 1 with a square-wave input of amplitude $\pm 1 \mathrm{~V}$ and frequency 1 kHz. First, we expand the state variable $V_{\text {OUT }}$ by Fourier series. Table I gives the relative simulation errors when different basis function numbers are employed. The relative error is defined as

$$
\operatorname{Err}_{R}=\sqrt{\frac{\int\left[y_{\mathrm{SPICE}}(t)-y_{\mathrm{Four}}(t)\right]^{2} d t}{\int\left[y_{\mathrm{SPICE}}(t)\right]^{2} d t}}
$$

where $y_{\text {SPICE }}(t)$ represents the exact steady-state response obtained by SPICE and $y_{\text {Four }}(t)$ represents the simulation response by the Fourier expansion. Note that the state equation should be integrated by SPICE
TABLE II

SimULATION RESUltS OF THE WAVELET EXPANSION (Without ADAPTIVE SCHEME) FOR MOS AMPLIFIER

\begin{tabular}{c|c|c}
\hline $\begin{array}{c}\text { Wavelet Space } \\
\text { Level } J\end{array}$ & $\begin{array}{c}\text { Overall Basis } \\
\text { Function Number }\end{array}$ & $\begin{array}{c}\text { Relative Simulation } \\
\text { Error for } V_{\text {ouT }}\end{array}$ \\
\hline 0 & 23 & $9.731733 \times 10^{-2}$ \\
1 & 43 & $5.427637 \times 10^{-2}$ \\
2 & 83 & $2.770902 \times 10^{-2}$ \\
3 & 163 & $1.328406 \times 10^{-2}$ \\
4 & 323 & $5.888023 \times 10^{-3}$ \\
5 & 643 & $2.250459 \times 10^{-3}$ \\
\hline
\end{tabular}

TABLE III

SimUlation Results of THE WaVElET EXPANSION (With AdAPTIVE SCHEME) FOR MOS AMPLIFIER

\begin{tabular}{c|c|c}
\hline $\begin{array}{c}\text { Wavelet Space } \\
\text { Level } J\end{array}$ & $\begin{array}{c}\text { Overall Basis } \\
\text { Function Number }\end{array}$ & $\begin{array}{c}\text { Relative Simulation } \\
\text { Error for } V_{\text {ouT }}\end{array}$ \\
\hline 0 & 23 & $9.731733 \times 10^{-2}$ \\
1 & 43 & $5.427637 \times 10^{-2}$ \\
2 & 77 & $2.770964 \times 10^{-2}$ \\
3 & 123 & $1.329575 \times 10^{-2}$ \\
4 & 195 & $5.933110 \times 10^{-3}$ \\
5 & 319 & $2.440557 \times 10^{-3}$ \\
6 & 515 & $1.233289 \times 10^{-3}$ \\
\hline
\end{tabular}

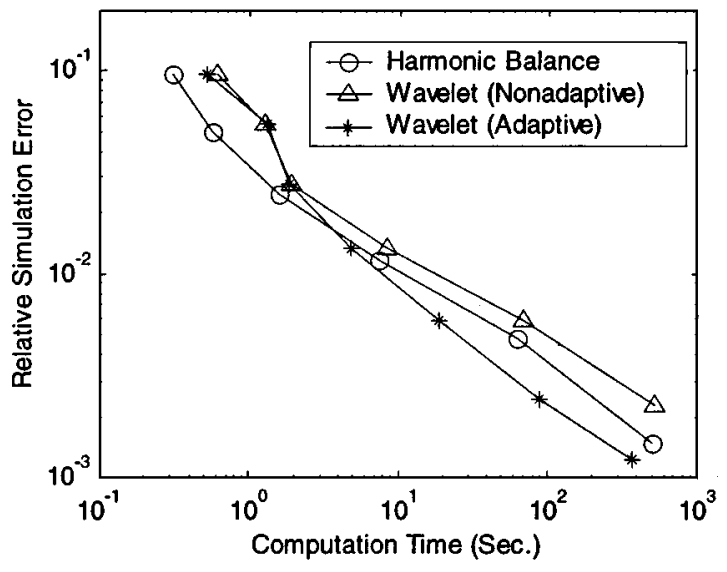

Fig. 2. Simulation results for MOS amplifier.

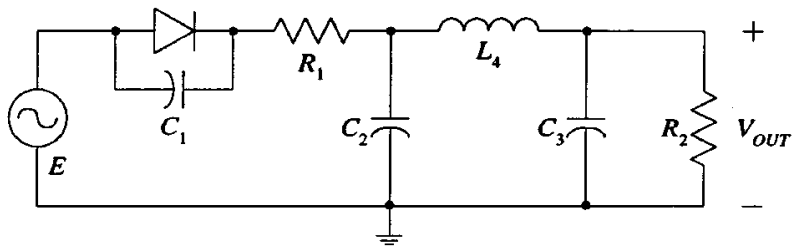

Fig. 3. Power supply where $C_{1}=1 \mu \mathrm{F}, C_{2}=C_{3}=1 \mathrm{mF}, L_{4}=0.1$ $\mathrm{H}, \boldsymbol{R}_{1}=\mathbf{5} \boldsymbol{\Omega}$ and $\boldsymbol{R}_{2}=1 \mathrm{~K} \boldsymbol{\Omega}$.

for quite a long time until the transients die out, so that an accurate steady-state response can be obtained.

Second, we expand the state variables by wavelets and apply the wavelet-balance method to calculate the steady-state response. When the adaptive technique is not used, all basis functions in given wavelet spaces are employed for computation. Table II gives the relative simulation errors under different wavelet basis function numbers. Comparing Table II with Table I, one would find that the wavelet-balance method 
TABLE IV

SIMULATION RESULTS OF THE FOURIER EXPANSION FOR POWER SUPPLY

\begin{tabular}{|c|c|c|c|c|c|c|}
\hline \multicolumn{5}{|c|}{ Basis Function Number } & \multirow{2}{*}{$\begin{array}{l}\text { Relative Simulation } \\
\text { Error for } V_{\text {out }}\end{array}$} & \multirow{2}{*}{$\begin{array}{l}\text { Computation Time } \\
\text { (Sec.) }\end{array}$} \\
\hline$V_{C 1}$ & $V_{C 2}$ & $V_{C 3}$ & $I_{L 4}$ & Total & & \\
\hline 11 & 11 & 11 & 11 & 44 & $8.058812 \times 10^{-3}$ & 0.380 \\
\hline 21 & 21 & 21 & 21 & 84 & $1.545737 \times 10^{-3}$ & 0.681 \\
\hline 41 & 41 & 41 & 41 & 164 & $3.086698 \times 10^{-4}$ & 1.512 \\
\hline 61 & 61 & 61 & 61 & 244 & $1.563388 \times 10^{-4}$ & 4.646 \\
\hline
\end{tabular}

TABLE V

Simulation Results of the WaVElet Expansion (With Adaptive Scheme) For POWER SuPPLy

\begin{tabular}{|c|c|c|c|c|c|c|c|}
\hline \multirow{2}{*}{$\begin{array}{l}\text { Wavelet Space } \\
\text { Level } J\end{array}$} & \multicolumn{5}{|c|}{ Basis Function Number } & \multirow{2}{*}{$\begin{array}{c}\text { Relative Simulation } \\
\text { Error for } V_{\text {OUT }}\end{array}$} & \multirow{2}{*}{$\begin{array}{c}\text { Computation Time } \\
\text { (Sec.) }\end{array}$} \\
\hline & $V_{C l}$ & $V_{C 2}$ & $V_{C 3}$ & $I_{L 4}$ & Total & & \\
\hline 0 & 13 & 13 & 13 & 13 & 52 & $8.086976 \times 10^{-3}$ & 0.410 \\
\hline 1 & 23 & 23 & 23 & 23 & 92 & $8.808400 \times 10^{-3}$ & 0.851 \\
\hline 2 & 31 & 29 & 23 & 31 & 114 & $1.420685 \times 10^{-4}$ & 1.161 \\
\hline 3 & 37 & 29 & 23 & 43 & 132 & $5.251310 \times 10^{-5}$ & 1.873 \\
\hline
\end{tabular}

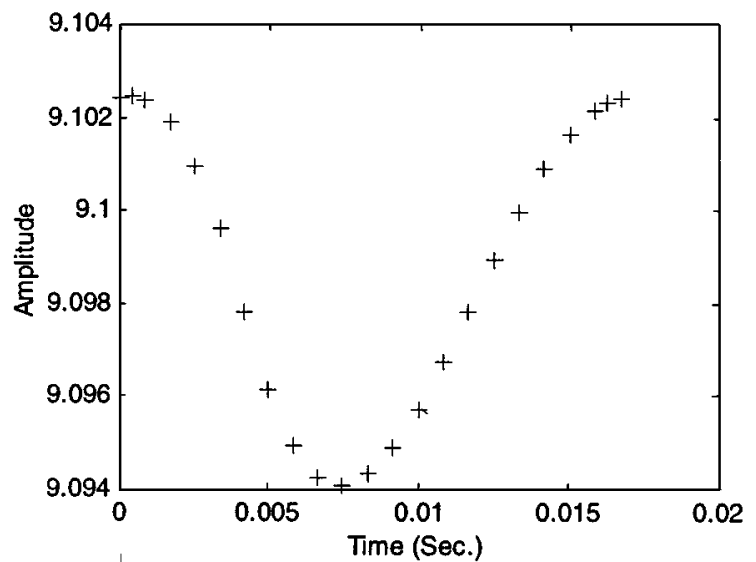

Fig. 4. Steady-state response of $\boldsymbol{V}_{\text {OUT }}$ in power supply.

hasn't gained any point in this example. The convergence rate of the Fourier expansion is a little higher than that of the wavelet approach.

Third, we show that the wavelet-balance method can improve its efficiency significantly after the adaptive algorithm is applied. Let the error tolerance $\varepsilon=10^{-3}$ and employ the adaptive technique to select proper basis functions automatically. Seven wavelet spaces (from level 0 to level 6) in all are applied by the adaptive algorithm during the course of iteration. Table III displays the relative simulation error and the total number of selected wavelet basis functions as different wavelet spaces are included. Two comments can be made according to the data in Tables I-III.

1) After the adaptive algorithm is applied, the number of wavelet bases which are used at a given space level is reduced since not all basis functions in that wavelet space are needed for computation. On the other hand, the simulation error for given space level $J$ doesn't change much as shown in Tables II and III. This demonstrates that the adaptive algorithm has the potential to pick up the most important basis functions and those ones neglected by the adaptive scheme don't contribute a lot to the simulation accuracy.

2) Taking advantage of the property of compact support, the adaptive algorithm can automatically select proper wavelet basis functions. However, Fourier bases have global support and it is infeasible to realize such a selection in time domain as

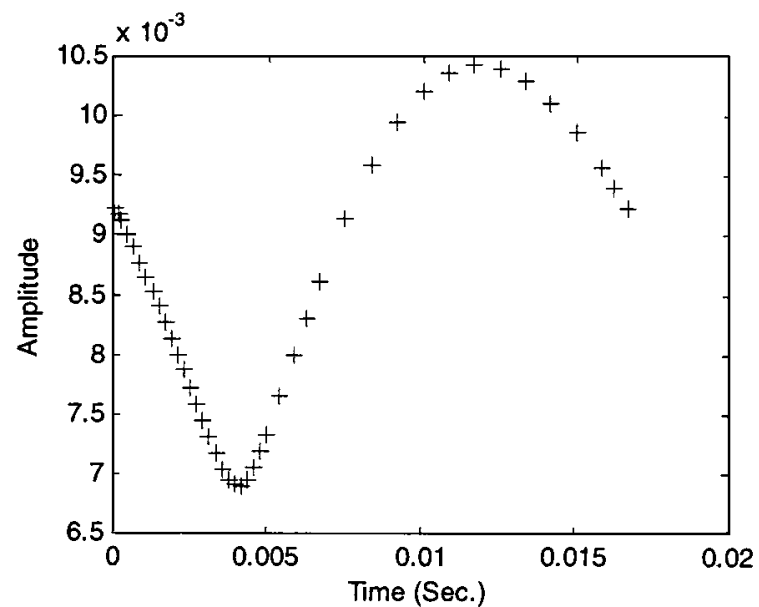

Fig. 5. Steady-state response of $\boldsymbol{I}_{L 4}$ in power supply.

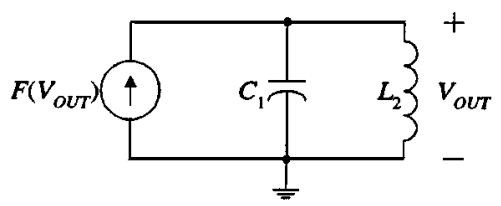

Fig. 6. Van der Pol oscillator where $C_{1}=1 \mathrm{~F}, L_{2}=1 \mathrm{H}$ and $\boldsymbol{F}\left(\boldsymbol{V}_{\text {OUT }}\right)=\mathbf{5} \cdot\left(\boldsymbol{V}_{\text {OUT }}-V_{\text {OUT }}^{3} / \mathbf{3}\right)$.

wavelets. Therefore, as displayed in Tables I and III, the wavelet expansion exhibits higher convergence rate than the Fourier approach after the adaptive scheme is employed.

Finally, Fig. 2 depicts the relation between simulation accuracy and computational time for the above three different approaches. Note that under small basis function numbers, the wavelet-balance method is not so efficient as the Fourier approach because almost all low-level wavelet basis functions are employed in order to capture those globalized components of the circuit response. However, as the wavelet space-level increases, the adaptive scheme begins to take effect and high level wavelet bases are only used in those regions where the circuit response exhibits localized singularities. Therefore, under large basis function numbers, the wavelet-balance method begins to catch up with the Fourier approach in accuracy and become more efficient. 
TABLE VI

SimUlation RESUlts OF THE FOURIER EXPANSION FOR VAN DER POL OSCILLATOR

\begin{tabular}{l|c|c|c|c}
\hline \multicolumn{3}{|c|}{ Basis Function Number } & \multirow{2}{*}{$\begin{array}{c}\text { Relative Simulation } \\
\text { Error for } V_{\text {OUT }}\end{array}$} & $\begin{array}{c}\text { Computation Time } \\
\text { (Sec.) }\end{array}$ \\
\hline$V_{C 1}$ & $I_{L 2}$ & Total & $4.963203 \times 10^{-1}$ & 0.981 \\
11 & 11 & 22 & $2.722372 \times 10^{-1}$ & 1,803 \\
21 & 21 & 42 & $1.119378 \times 10^{-1}$ & 2.203 \\
41 & 41 & 82 & $6.799617 \times 10^{-2}$ & 6.159 \\
61 & 61 & 122 & $6.744133 \times 10^{-2}$ & 8.482 \\
\hline
\end{tabular}

TABLE VII

Simulation Results of the WaVelet Expansion (With AdAPTive Scheme) For Van DER Pol Oscillator

\begin{tabular}{c|c|c|c|c|c}
\hline \multirow{2}{*}{$\begin{array}{c}\text { Wavelet Space } \\
\text { Level } J\end{array}$} & \multicolumn{3}{|c|}{ Basis Function Number } & $\begin{array}{c}\text { Relative Simulation } \\
\text { Error for } V_{\text {ouT }}\end{array}$ & $\begin{array}{c}\text { Computation Time } \\
\text { (Sec.) }\end{array}$ \\
\cline { 2 - 4 } & $V_{C 1}$ & $I_{L 2}$ & Total & $4.885475 \times 10^{-1}$ & 0.962 \\
1 & 13 & 13 & 26 & $2.617620 \times 10^{-1}$ & 2.223 \\
2 & 23 & 23 & 46 & $1.298582 \times 10^{-1}$ & 2.452 \\
3 & 41 & 35 & 76 & $7.386915 \times 10^{-2}$ & 2.784 \\
4 & 57 & 55 & 112 & $5.622640 \times 10^{-2}$ & 6.880 \\
\hline
\end{tabular}

\section{B. Power Supply}

The power-supply circuit shown in Fig. 3 is one of the several examples that has been used in the past to test various steady-state analysis algorithms for nonautonomous circuits [1], [2], [4], [7]. The input excitation in Fig. 3 is a standard sinusoidal signal of amplitude $\pm 10 \mathrm{~V}$ and frequency $60 \mathrm{~Hz}$. First, we expand the state variables $V_{C 1}, V_{C 2}, V_{C 3}$ and $I_{L 4}$ by Fourier series. Table IV displays the simulation errors and computation times when different basis function numbers are used. Second, we expand the state variables by wavelets and apply the adaptive scheme to automatically choose necessary wavelet basis functions with error tolerance $\varepsilon=10^{-3}$. Table $\mathrm{V}$ gives the simulation results obtained by the wavelet-balance method. The effect of the adaptive algorithm is well illustrated by Tables IV and V that the wavelet expansion overtakes the Fourier technique in efficiency as the basis function number increases.

Figs. 4 and 5 depict the steady-state response $V_{\text {OUT }}$ and $I_{L 4}$ obtained by the wavelet-balance method at space level $J=3$. As indicated by the distribution of collocation points ${ }^{1}$, the wavelet basis functions which are adaptively employed for representing $I_{L 4}$ are much more than those for $V_{\mathrm{OUT}}$, since the waveform of $I_{L 4}$ is more singular than that of $V_{\text {OUT }}$. In addition, most wavelet basis functions in Fig. 5 concentrate in interval $[0,0.005]$ where the response of $I_{L 4}$ exhibits a fast transition. The above comparison demonstrates that the waveforms with different singularities are treated by the adaptive scheme differently. High-level wavelet bases are only used near sharp transitions, which is exactly the reason why the wavelet-balance method is more efficient than the Fourier approach in this example.

\section{Van der Pol Oscillator}

Because of the high nonlinearity, the Van der Pol oscillator is served as a benchmark circuit for various steady-state algorithms for autonomous circuits, such as those in [4], [8], [9]. The circuit schematic is quite simple as illustrated in Fig. 6. Tables VI and VII display the simulation results obtained by the Fourier expansion and

${ }^{1}$ Each wavelet basis function is combined with a collocation point, and the wavelet basis function is localized in the neighborhood of its corresponding collocation point. Therefore, the distribution of collocation points reflects the distribution of wavelet basis functions.

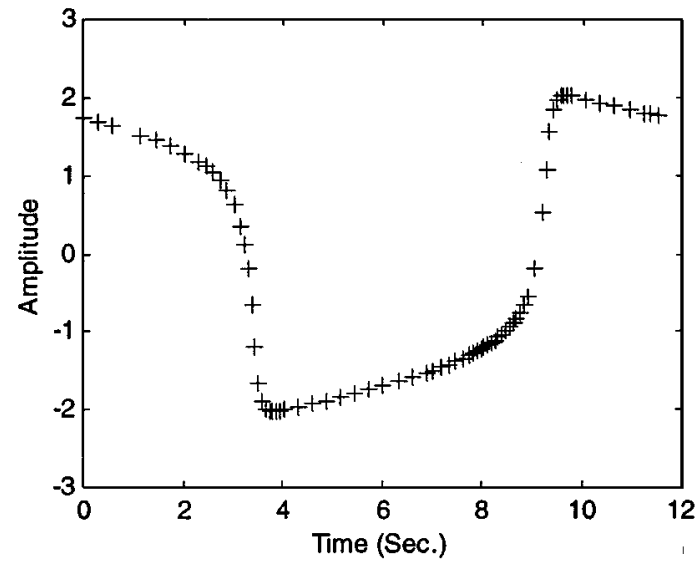

Fig. 7. Steady-state response of $\boldsymbol{V}_{\text {OUT }}$ in Van der Pol oscillator.

the wavelet-balance method (with adaptive scheme at error tolerance $\varepsilon=10^{-2}$ ) respectively. Again, the wavelet-balance method reveals its effectiveness in both speed and accuracy when solving the steady-state problems.

Fig. 7 depicts the output waveform $V_{\text {OUT }}$ simulated by the waveletbalance method at space level $J=4$. Note that the adaptive scheme only automatically employ high level basis functions in interval [2, 5] and $[7,10]$, where fast transitions take place.

\section{CONCLUSION REMARKS}

We present in this brief, a novel wavelet-balance method for steadystate analysis of nonlinear circuits. As a counterpart of those Fourierbased techniques, the wavelet-balance method works in time domain and has a solid theoretical background based on the wavelet approximation theory. Taking advantage of the superior computational properties of wavelets, the proposed method can automatically select proper basis functions for computation so that it presents greater efficiency than the conventional Fourier-based techniques in many practical problems. From this point of view, the wavelet-balance method exploits a new approach to access the steady-state problem besides those frequency domain methods that have been used for a long time. 


\section{APPENDIX}

In this Appendix, we briefly present some important background of the wavelet basis functions used in this paper.

In Sobolev space $H^{2}[0, L]$, we first introduce the following function subspaces:

$$
\begin{aligned}
V_{-1} & =\operatorname{span}\left\{\begin{array}{c}
\eta_{1}(t), \eta_{2}(t), \eta_{1}(L-t), \eta_{2}(L-t), \varphi_{0,-1}(t) \\
\varphi_{0,0}(t), \varphi_{0,1}(t), \ldots, \varphi_{0, L-4}(t), \varphi_{0, L-3}(L-t)
\end{array}\right\} \\
W_{J} & =\operatorname{span}\left\{\Psi_{J, K}(t),-1 \leq K \leq 2^{J}-2\right\}, \quad J \geq 0 \\
V_{J} & =V_{J-1}+W_{J}, \quad J \geq 0
\end{aligned}
$$

where the definitions of functions $\eta_{1}(t), \eta_{2}(t), \varphi_{0,-1}(t),\left\{\varphi_{0, K}(t)\right.$, $0 \leq K \geq L-4\}, \varphi_{0, L-3}(L-t)$ and $\left\{\Psi_{J, K}(t),-1 \leq K \leq\right.$ $\left.2^{J}-2, J \geq 0\right\}$ are given below. $\operatorname{span}\left\{f_{1}, f_{2}, \ldots, f_{N}\right\}$ is a function set formed by all linear combinations of the functions $f_{1}, f_{2}, \ldots, f_{N}$.

In (A.1), the function $\eta_{1}(t), \eta_{2}(t)$ are used to handle nonhomogeneity of the boundary data

$$
\begin{aligned}
& \eta_{1}(t)=(1-t)_{+}^{3} \\
& \eta_{2}(t)=2 t_{+}-2 t_{+}^{2}+\frac{7}{6} t_{+}^{3}-\frac{4}{3}(t-1)_{+}^{3}+\frac{1}{6}(t-2)_{+}^{3}
\end{aligned}
$$

where

$$
t_{+}^{n}= \begin{cases}t^{n}, & \text { if } t \geq 0 \\ 0, & \text { otherwise }\end{cases}
$$

The boundary scaling functions are

$$
\begin{aligned}
\varphi_{0,-1}(t)= & \varphi_{b}(t)=\frac{3}{2} t_{+}^{2}-\frac{11}{12} t_{+}^{3}+\frac{3}{2}(t-1)_{+}^{3} \\
& -\frac{3}{4}(t-2)_{+}^{3}+\frac{1}{6}(t-3)_{+}^{3} \\
\varphi_{0, L-3}(t)= & \varphi_{b}(L-t)
\end{aligned}
$$

and the interior scaling functions

$$
\varphi_{0, K}(t)=\varphi(t-K), \quad 0 \leq K \leq L-4
$$

where

$$
\begin{aligned}
\varphi(t)=\frac{1}{6} t_{+}^{3}-\frac{2}{3}(t-1)_{+}^{3}+(t-2)_{+}^{3} & \\
& -\frac{2}{3}(t-3)_{+}^{3}+\frac{1}{6}(t-4)_{+}^{3} .
\end{aligned}
$$

The boundary wavelet functions are

$$
\begin{aligned}
\psi_{J,-1}(t) & =\psi_{b 0}\left(2^{J} t\right), \quad \psi_{J, 0}(t)=\psi_{b 1}\left(2^{J} t\right) \\
\psi_{J, 2} J_{-3} & (t) \\
\psi_{J, 2 J-2}(t) & =\psi_{b 1}\left[2^{J}(L-t)\right]
\end{aligned}
$$

where

$$
\begin{aligned}
& \psi_{b 0}(t)=-\frac{56}{99}[14 \psi(t+2)+\psi(t+1)] \\
& \psi_{b 1}(t)=-\frac{182}{181}\left[\psi(t)+\frac{1}{13} \psi(t+1)+\frac{1}{13} \psi(t+2)\right]
\end{aligned}
$$

and the interior wavelet functions are

$$
\psi_{J, K}(t)=\psi\left(2^{J}-K\right), \quad 1 \leq K \leq 2^{J}-4
$$

where

$$
\psi(t)=-\frac{3}{7} \varphi(2 t)+\frac{12}{7} \varphi(2 t-1)-\frac{3}{7} \varphi(2 t-2) .
$$

The total number of basis functions in subspace $V_{J}$ is $N=2^{J+1} L+3$.

On the other hand, the following collocation points are chosen for $V_{-1}$ and $W_{J}, J \geq 0$, respectively

$$
\begin{cases}t=0, \frac{1}{2}, 1,2, \ldots, L-1, L-\frac{1}{2}, L, & \text { for } V_{-1} \\ t=\frac{1}{2^{J+2}}, \frac{1.5}{2^{J}}, \frac{2.5}{2^{J}}, \ldots, \frac{2^{J}-1.5}{2^{J}}, L-\frac{1}{2^{J+2}}, & \text { for } W_{J}\end{cases}
$$

The above content only gives a simple outline of the wavelet basis functions and collocation points used in this paper, and further details can be found in [15]-[17].

\section{ACKNOWLEDGMENT}

The authors wish to thank Prof. D. Zhou, University of Texas, Dallas for the helpful discussions, and all the anonymous reviewers of this paper for their valuable suggestions.

\section{REFERENCES}

[1] T. J. Aprille Jr. and T. N. Trick, "Steady-state analysis of nonlinear circuits with periodic inputs," Proc. IEEE, vol. 60, pp. 108-114, Jan. 1972.

[2] S. Skelboe, "Computation of the periodic steady-state response of nonlinear circuits by extrapolaton methods," IEEE Trans. Circuits Syst., vol. 27, pp. 161-175, Mar. 1980.

[3] M. Kakizaki and T. Sugawara, "A modified Newton method for the steady-state analysis," IEEE Trans. Computer-Aided Design, vol. 4, pp. 662-667, Oct. 1985.

[4] J. R. Parkuhurst and L. L. Ogborn, "Determining the steady-state output of nonlinear oscillatory circuits using multiple shooting," IEEE Trans. Computer-Aided Design, vol. 14, pp. 882-889, July 1995.

[5] A. Ushida and L. O. Chua, "Frequency-domain analysis of nonlinear circuits driven by multi-tone signals," IEEE Trans. Circuits Syst., vol. 31, pp. 766-779, Sept. 1984.

[6] K. S. Kundert and A. Sangiovanni-Vincentelli, "Simulation of nonlinear circuits in the frequency domain," IEEE Trans. Computer-Aided Design, vol. 5, pp. 521-535, Oct. 1986.

[7] D. R. Frey and O. Norman, "An integral equation approach to the periodic steady-state problem in nonlinear circuits," IEEE Trans. Circuits Syst. I, vol. 39, pp. 744-755, Sept. 1992.

[8] P. Pala-Schonwalder and J. M. Miro-Sans, "A discrete time approach to the steady state analysis of nonlinear autonomous circuits," Int. J. Circuit Theory Appl., vol. 23, no. 6, pp. 297-310, 1994.

[9] D. R. Frey, "A class of relaxation algorithms for finding the periodic steady-state solution in nonlinear system," IEEE Trans. Circuits Syst. I, vol. 45, pp. 659-663, June 1998.

[10] Y. Yamagami, Y. Nishio, A. Ushida, M. Takahashi, and K. Ogawa, "Analysis of communication circuits based on multidimensional Fourier transformation," IEEE Trans. Computer-Aided Design, vol. 18, pp. 1165-1177, Aug. 1999

[11] F. Filicori, M. R. Scalas, and C. Naldi, "Nonlinear circuit analysis through periodic spline approximation," Electron. Lett., vol. 15, no. 19, pp. 597-599, 1979.

[12] Y. Meyer, Ondelettes ét opétateurs, I: Ondelettes. Paris, France: Hermann, 1990.

[13] C. K. Chui, An Introduction to Wavelets. New York: Academic, 1992.

[14] E. Bacry, S. Mallat, and G. Papanicolaou, "A wavelet based space-time adaptive numerical method for partial differential equations," Math. Model. Numer. Anal., vol. 26, no. 7, pp. 793-834, 1992.

[15] W. Cai and J. Z. Wang, "Adaptive multi-resolution collocation methods for initial boundary value problems of nonlinear PDE's," SIAM J. Numer. Anal., vol. 33, no. 3, pp. 937-970, 1996.

[16] D. Zhou and W. Cai, "A fast wavelet collocation method for high-speed circuit simulation," IEEE Trans. Circuits Syst. I, vol. 46, pp. 920-930, Aug. 1999.

[17] D. Zhou, W. Cai, and W. Zhang, "An adaptive wavelet method for nonlinear circuit simulation," IEEE Trans. Circuits Syst. I, vol. 46, pp. 931-938, Aug. 1999

[18] D. Marquardt, "An algorithm for least-squares estimation of nonlinear parameters," SIAM J. Appl. Math., vol. 11, no. 2, pp. 431-441, 1963. 